\title{
MicroRNA-490-5p is a novel tumor suppressor targeting c-FOS in human bladder cancer
}

\author{
Gongbin Lan ${ }^{1}$, Luoyan Yang ${ }^{2}$, Xubiao Xie ${ }^{1}$, Longkai Peng ${ }^{1}$, Yi Wang ${ }^{2}$
}

${ }^{1}$ Department of Kidney Transplantation, The Second Xiangya Hospital of Central South University, Changsha, China

${ }^{2}$ Department of Urology, The Second Xiangya Hospital of Central South University, Changsha, China

Submitted: 2 February 2013

Accepted: 25 July 2013

Arch Med Sci 2015; 11, 3: 561-569

DOI: 10.5114/aoms.2015.52359

Copyright $\odot 2015$ Termedia \& Banach

\section{Abstract}

Introduction: Recent studies have demonstrated the critical roles of microRNAs in tumorigenesis and tumor progression. Here, we describe the regulation and function of miR-490-5p in bladder cancer.

Material and methods: Paired tissue samples were collected from bladder cancer patients $(n=20)$. Real-time PCR revealed that miR-490-5p expression was significantly down-regulated in human bladder cancer tissues and cells. Also there was an inverse relationship between the expression level of miR490-5p and the pathological grade of bladder cancer. Western blotting was performed to detect the expression levels of c-FOS and TET1 in 6 matched tumor tissue samples and 4 bladder cell lines. Furthermore, to better understand the underlying mechanisms of miR-490-5p, we conducted gain and loss of function analysis by transfecting bladder cancer T24 cells with chemically synthesized miR-490-5p mimics and inhibitor, respectively.

Results: We found that overexpression of miR-490-5p in T24 cells could inhibit cell proliferation and invasion and induce cell apoptosis. Conversely, suppression of miR-490-5p expression induced cell proliferation and invasion, while it inhibited cell apoptosis. In addition, our bioinformatics prediction and experimental data showed that c-FOS was a potential target of miR-490-5p. The expression level of c-FOS was significantly decreased after miR-490-5p overexpression and significantly increased after miR-490-5p suppression, indicating that c-FOS was a target of miR-490-5p.

Conclusions: These findings suggest that miR-490-5p is a novel tumor suppressor, contributing to the carcinogenesis of bladder cancer by targeting c-FOS.

Key words: bladder cancer, miR-490-5p, tumor suppressor, c-FOS.

\section{Introduction}

Bladder cancer is one of the most common malignancies worldwide. According to the global cancer statistics published in 2011 [1], there are an estimated 386,300 new cases diagnosed and 150,200 deaths occurred in 2008. Currently, there are many therapeutic strategies for the treatment of bladder cancer, such as surgery, intravesical instillation therapy and radiation therapy [2-4]. However, patients with advanced bladder cancer still face a low survival rate with a 5 -year survival rate of $20-40 \%$ [5-7]. There are several factors affecting the prognosis of an invasive bladder tumor patient with radical cystectomy, including tumor grade, tumor stage, preoperative uremia and lymph node involvement [8].

\author{
Corresponding author: \\ Longkai Peng \\ Department of Kidney \\ Transplantation \\ The Second Xiangya Hospital \\ of Central South University \\ Changsha, 410011, China \\ Phone: +8673185295141 \\ Fax: +86 73185292191 \\ E-mail: plk2012@163.com
}


Therefore it is urgent to study the possible factors that trigger the tumorigenesis of bladder cancer and to develop novel anti-cancer strategies.

Many genetic and environmental factors contribute greatly to the carcinogenesis of bladder cancer through the activation of oncogenes and invalidation of cancer suppressor genes [9]. For example, some researchers found that hypermethylation of promoter regions in the p16 and DAPK genes was closely related to the process of bladder cancer genesis [10]. With the rapid development of advanced genomic sequencing technology, accumulating evidence supports the supposition that miRNAs may play critical roles in cancer development through regulating cellular activities such as proliferation, apoptosis and differentiation [1113]. Therefore, it is desirable to develop an anticancer strategy that targets miRNAs based on their functions and underlying mechanisms.

MicroRNAs (miRNAs) are an abundant class of short (about 22 nucleotides in length) and highly conserved small noncoding RNAs molecules, which act as negative regulators of gene expression by binding to specific complementary messenger RNAs $[14,15]$. It has been estimated that $30 \%$ of human genes are regulated by miRNAs [16]. According to computational predictions, a single miRNA can regulate nearly 200 target genes [17] Some miRNAs function as oncogenes, while others function as tumor suppressor genes in cancers [18]. Aberrant miRNA expression has been observed in bladder cancer, such as the up-regulation of miR21, miR-96 and miR-183, and the down-regulation of miR-143, miR-145 and miR-1826 [19-22]. Recently, a study reported that miR-490-5p was significantly down-regulated in bladder cancer compared to matched histologically normal urothelium, suggesting its participation in tumor progression [23]. However, to date, the underlying mechanism of miR-490-5p in bladder cancer remains unknown.

In the present research, the expression of miR490-5p was significantly down-regulated in bladder cancer tissues and an inverse correlation of miR-490-5p with pathological classifications was observed. Also the expression of miR-490-5p was significantly decreased in three bladder cancer cell lines (T24, BIU-87 and 5637) compared with a normal bladder cell line (SV-HUC-1). Additionally, we demonstrated that miR-490-5p could function as a tumor suppressor in human bladder cancer T24 cells by targeting c-FOS.

\section{Material and methods}

\section{Tissue samples and cell lines}

The paired tissue samples were collected from bladder cancer patients $(n=20)$ who had received cystectomy in the Second Xiangya Hospital between 2008 and 2010. These tissues were stored in liquid nitrogen. The cancer samples were staged according to the bladder cancer grading system of the World Health Organization (WHO) published in 1973. These cancer samples included 9 grade I and 11 grade II-III samples. Our study was approved by the Institutional Ethics Committee of Central South University. Patients enrolled were well informed and consent was obtained.

The human bladder cancer cell lines (T24, BIU-87 and 5637) and immortalized human uroepithelial cell line (SV-HUC-1) were purchased from Shanghai GenePharma Technology Co., Ltd (Shanghai, China). The cell lines were maintained at a $37^{\circ} \mathrm{C}$ in a $5 \% \mathrm{CO}_{2}$ incubator in DMEM (Sigma, America) supplemented with $10 \%$ fetal bovine serum (FBS) (Gibco, America).

\section{Real-time polymerase chain reaction}

Real-time PCR assays were performed to detect the expression levels of miR-490-5p in bladder tissue samples and cell lines. Total RNAs were extracted from fresh tissues or cells with TRIzol reagent (Invitrogen, Carlsbad, CA, USA). Real-time PCR assays were performed using a miRNeasy mini kit (Qiagen, USA; cat no. 217004) and a miRNA Q-PCR detection kit (GeneCopoeia, Rockville, MD; USA, cat no. R0101L). The primers used were 5'-CATGGATCTCCAGGTGG-3' (sense) and 5'- TGGTGTCGTGGAGTCG-3' (anti-sense). The relative expression level of miR-490-5p was normalized to U6 by $2^{-\Delta \Delta C t}$ methods [24]. Experiments were performed three times.

\section{Target gene prediction}

Target genes of miR-490-5 $p$ were predicted using software including iRBase [25], microRNA and TargetScan. The two target genes predicted were c-FOS and Ten-Eleven-Translocation 1 (TET1).

\section{Western blotting}

Western blotting was performed to detect the expression levels of c-FOS and TET1 in 6 matched tumor tissue samples and 4 bladder cell lines. The effect of miR-490-5p on the expression of c-FOS was also detected by western blotting analysis. The primary antibodies used were: c-FOS (cat no. sc-447; Santa Cruz Biotechnology, Santa Cruz, CA, USA), Ten-Eleven-Translocation 1 (TET1) (cat no. sc-163443; Santa Cruz Biotechnology, Santa Cruz, (A, USA) and glyceraldehyde 3-phosphate dehydrogenase (GAPDH) (cat no. Mab-2005079; ProMab). Each experiment was repeated more than 3 times. Quantification of c-FOS and TET1 protein levels was determined using the Image software. Relative expression levels of c-FOS and TET1 were normalized to GAPDH and presented as fold change relative to the level of control. 


\section{Cell transfection}

The day before transfection, 4-5 × 104 T24 cells were seeded in 6-well plates. The transfection of miR-490-5p mimics (or inhibitors) and negative control miRNA mimics (chemically synthesized by GenePharma, Shanghai, China) was performed using Lipofectamine 2000 (Invitrogen, Carlsbad, CA) according to the manufacturer's protocol. The expression levels of miR-490-5p in both groups were determined after $48 \mathrm{~h}$ of transfection.

\section{MTT assay}

Cell viability was assessed by MTT assay. In brief, at $0 \mathrm{~h}, 24 \mathrm{~h}, 48 \mathrm{~h}$ and $72 \mathrm{~h}$ after transfection, T24 cells were seeded into 96 -well plates at a concentration of $1 \times 10^{4}$ cells per well. MTT $(50 \mu \mathrm{l})$ (KeyGEN, Nan Jing, China) was added to each well and incubated for $4 \mathrm{~h}$ at $37^{\circ} \mathrm{C}$. After removal of the supernatant, the formazan crystals were dissolved in $150 \mu \mathrm{l}$ DMSO (Sigma) and absorbance at $570 \mathrm{~nm}$ was measured.

\section{Cell apoptosis analysis}

Cell apoptosis was detected by assaying caspase-3 activity, which was measured by a caspase-3 Activity Colorimetric assay kit (KeyGEN, Nan Jing, China) following the manufacturer's instructions. Acetyl-Asp-Glu-Val-Asp p-nitroanilide (Ac-DEVD-pNA) was used as a substrate. The fluorescence of the released $\mathrm{pNA}$ was measured with an excitation wavelength of $405 \mathrm{~nm}$.
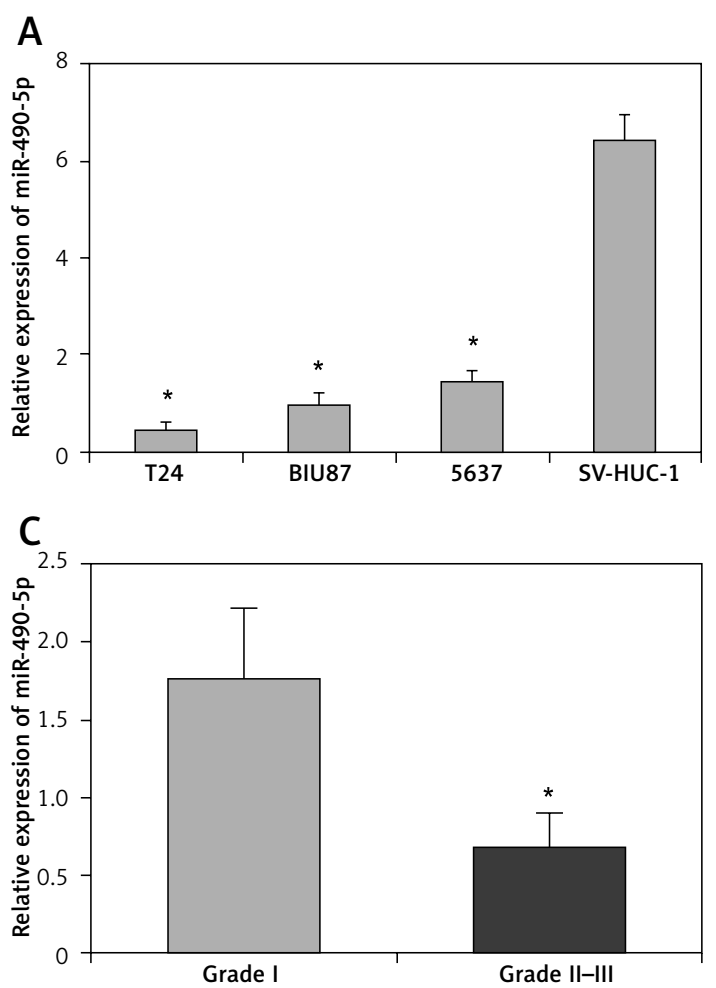

\section{Cell invasion assay}

Cell invasion assays were performed to detect the ability of cells passing through a Transwell chamber (Corning, NY, USA). At $48 \mathrm{~h}$ after transfection, cells were seeded on a Matrigel-coated membrane matrix in the insert of a 6-well culture plate and FBS was added to the lower chamber. After culturing for $24 \mathrm{~h}$, the lower surface of the Transwell chamber was observed by the inverted microscope (Olympus), and numbers of cells were counted.

\section{Statistical analysis}

The data were presented as mean \pm standard deviation $(\bar{x} \pm \mathrm{SD})$. The statistical analyses were performed by one-way LSD test or Student's $t$-test, respectively, using the SPSS 16.0 program. Value of $p<0.05$ was considered significantly different or correlated.

\section{Results}

\section{MiR-490-5p is down-regulated in human bladder cancer tissues and cell lines}

First, we analyzed the expression levels of miR490-5p in three human bladder cancer cell lines by real-time PCR. As shown in Figure $1 \mathrm{~A}$, the miR490-5p expression levels in three bladder cancer cell lines were significantly down-regulated compared with those in SV-HUC-1 cells. Next we detected the expression levels of miR-490-5p in 20 bladder cancer tissues and their paired adjacent

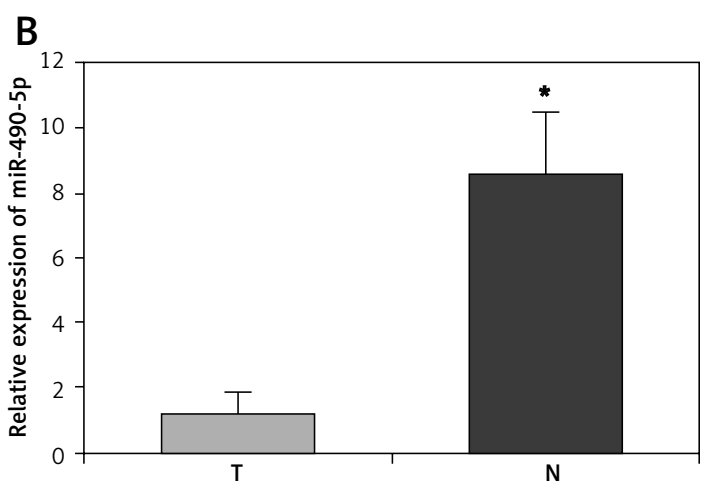

Figure 1. Relative expression levels of miR-490$5 \mathrm{p}$ in bladder cancer tissues and bladder cancer cell lines. Expression levels of miR-490-5p were examined by real-time PCR. A - Expression levels of miR-490-5p in SV-HUC-1 cells and three bladder cancer cell lines. One-way LSD test, ${ }^{*} p<0.01$. B - Expression levels of miR-490-5p in 20 paired bladder cancer and adjacent non-tumor tissues. Student's $t$-test, ${ }^{*} p<0.01$. C - Expression levels of miR-490-5p in different pathological grades. Student's $t$-test, ${ }^{*} p<0.01$

$T$ - Tumor tissues, $N$ - adjacent non-tumor tissues. 
non-tumor tissues. Consistent with the data obtained from bladder cancer cell lines, the average expression level of miR-490-5p was significantly lower in bladder cancer tissues than in paired adjacent non-tumor tissues (Figure $1 \mathrm{~B}$ ).

Also we assessed the expression levels of miR490-5p in bladder cancers of various pathological classifications. As shown in Figure $1 \mathrm{C}$, the expression level of miR-490-5p was significantly higher in pathological grade I than in grade II-III. This result suggested that there was an inverse correlation between the expression of miR-490-5p and the pathological classifications of bladder cancer.

A

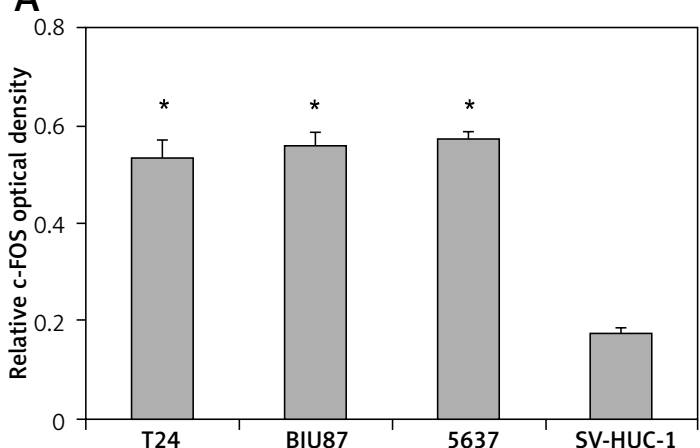

C

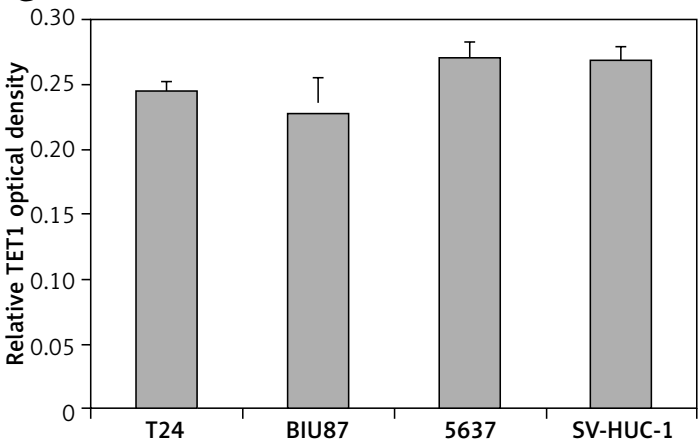

E
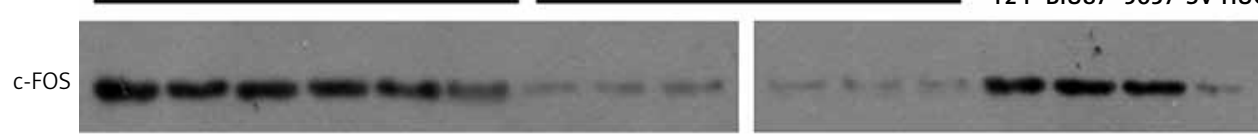

TET1

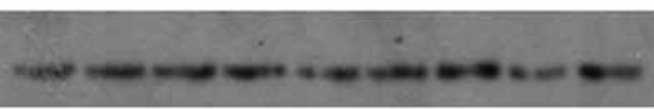

GAPDH

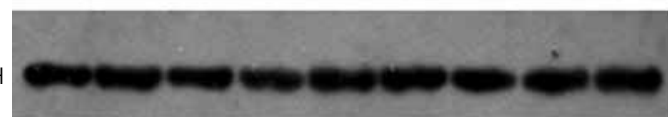

In short, the results indicate that the expression levels of miR-490-5p were significantly decreased in human bladder cancer tissues and cell lines. Thus, we speculate that miR-490-5p might be a potential tumor suppressor in bladder cancer. The T24 cell line was used for further research because it had the lowest miR-490-5p expression level among three bladder cancer cell lines.

\section{The protein level of c-FOS is up-regulated in bladder cancer tissues and cell lines}

Two genes, c-FOS and TET1, were predicted to be targets of miR-490-5p using miRNA analysis
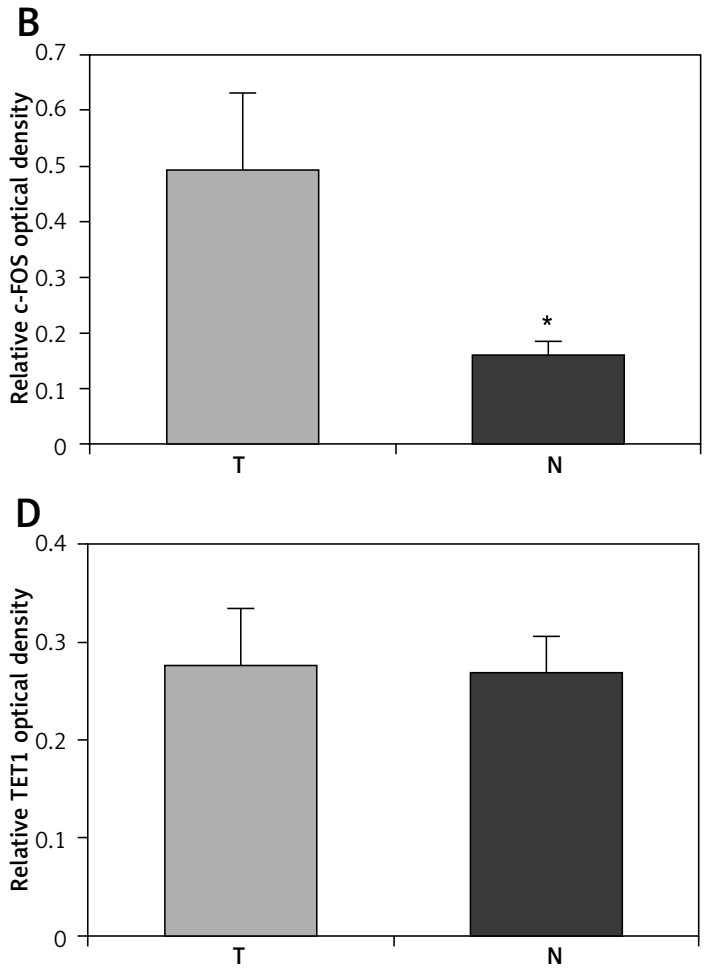

$\mathrm{N}$

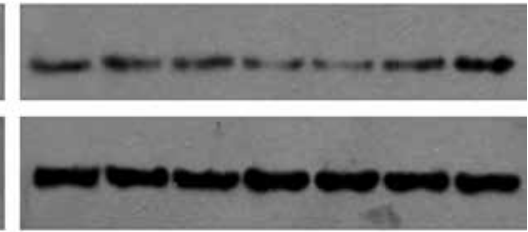

Figure 2. Relative expression levels of c-FOS and TET1 in bladder cancer tissues and bladder cancer cell lines. Quantitative results of western blotting are shown in Figures 2 A-D and representative western blotting results are shown in Figure 2 E. A - Mean level of c-FOS expression in three bladder cancer cell lines and SV-HUC-1 cells. One-way LSD test, ${ }^{*} p<0.01$. B - Mean level of c-FOS expression in six bladder cancer specimens and paired adjacent non-tumor tissues. Student's $t$-test, ${ }^{*} p<0.01$. C - TET1 expression levels in three bladder cancer cell lines and SV-HUC-1 cell line $(p>0.05)$. D - TET1 expression levels in six bladder cancer tissues and adjacent non-tumor tissues $(p>0.05)$. E - Relative levels of c-FOS and TET1 were examined by western blotting. GAPDH was used as an internal control

$T$ - Tumor tissues, $N$ - adjacent non-tumor tissues. 
software. Thus we analyzed the protein levels of c-FOS and TET1 in tumor tissues and tumor cell lines. Quantitative results of western blotting are shown in Figures $2 A-D$ and representative western blotting results are shown in Figure $2 \mathrm{E}$. As shown in Figures $2 \mathrm{~A}, \mathrm{E}$, the c-FOS protein levels in three bladder cancer cell lines were significantly higher compared with those in the SV-HUC-1 cell line. Consistent with these data, the average protein level of c-FOS was significantly up-regulated in 6 bladder cancer specimens compared to paired adjacent non-tumor tissues (Figures 2 B, E). However, there was no significant difference in TET1 expression levels in all three bladder cancer cell lines compared with those in the SV-HUC-1 cell line (Figures 2 C, E). Similarly, the TET1 expression levels between bladder cancer tissues and adjacent non-tumor tissues showed no significant difference (Figures 2 D, E), which suggested that miR-490-5p expression had no relationship with TET1 expression.

These results indicated that the miR-490-5p expression decrease was correlated with the c-FOS expression increase in bladder cancer tissues and bladder cancer cell lines.
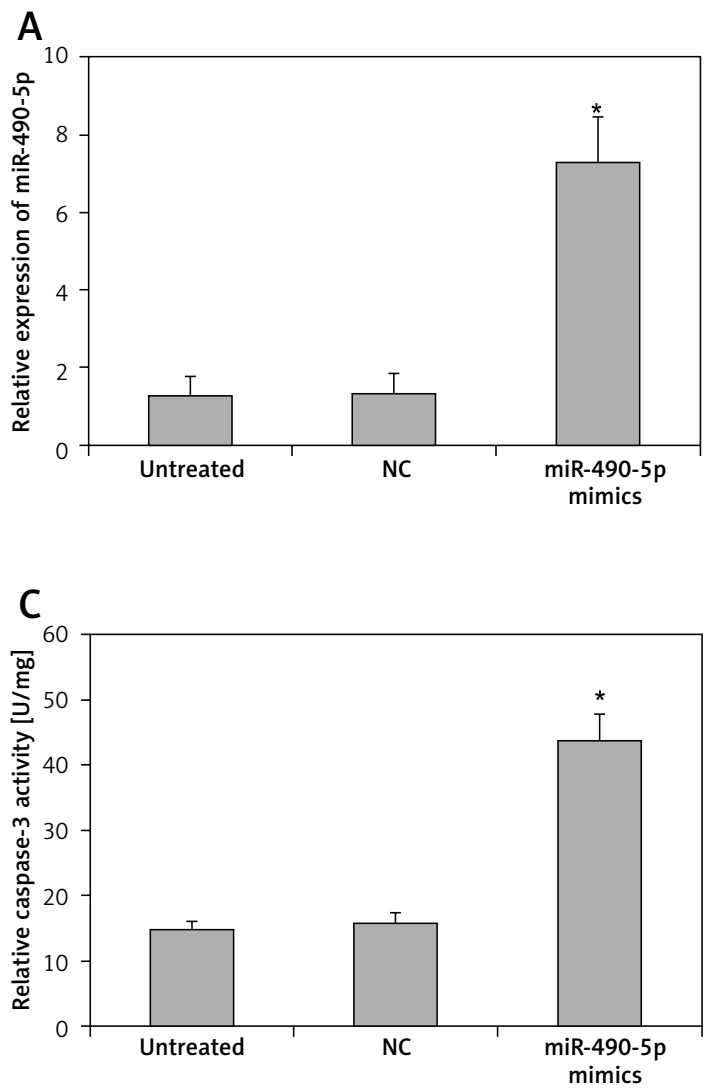

Effects of miR-490-5p up-regulation on cell proliferation, apoptosis and invasion activity of $\mathrm{T} 24$ cells

To determine whether miR-490-5p functions as a tumor suppressor, we transfected T24 cells with miR-490-5p mimics and evaluated the effects of miR-490-5p over-expression. First we measured the effects of the chemically synthesized miR-490-5p mimics on the expression of miR-490-5p by real-time PCR. As shown in Figure $3 \mathrm{~A}$, the expression level of miR-490-5p in the miR-490-5p mimics group was significantly higher than in the negative control miRNA group and the untreated group. This result indicates that miR-490-5p mimics were successfully transfected into the T24 cells.

Cell proliferation was determined by the MTT assay at $0 \mathrm{~h}, 24 \mathrm{~h}, 48 \mathrm{~h}$ and $72 \mathrm{~h}$ post-transfection. As shown in Figure $3 \mathrm{~B}$, at $\mathrm{O} \mathrm{h}$ and $24 \mathrm{~h}$ after transfection, there were no significant differences among the three groups. However at $48 \mathrm{~h}$ and $72 \mathrm{~h}$ after transfection, the miR-490-5p transfected cells exhibited significant growth inhibition compared with the negative control (miRNA transfected) cells or the untreated T24 cells.

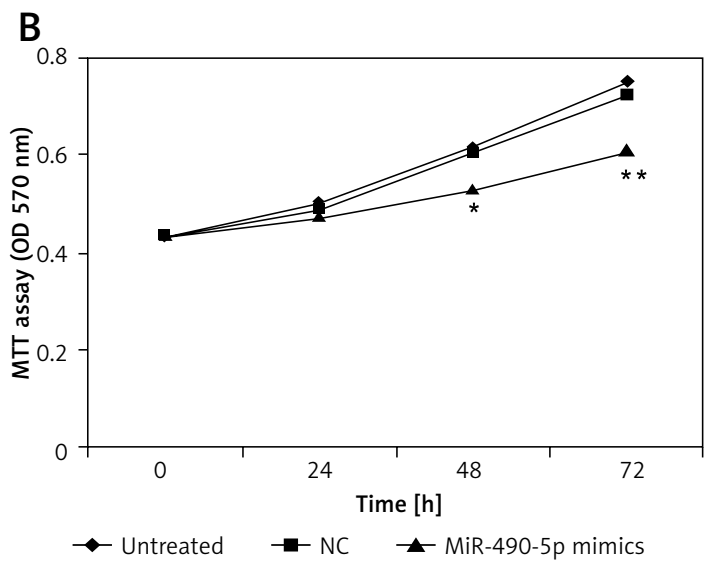

D

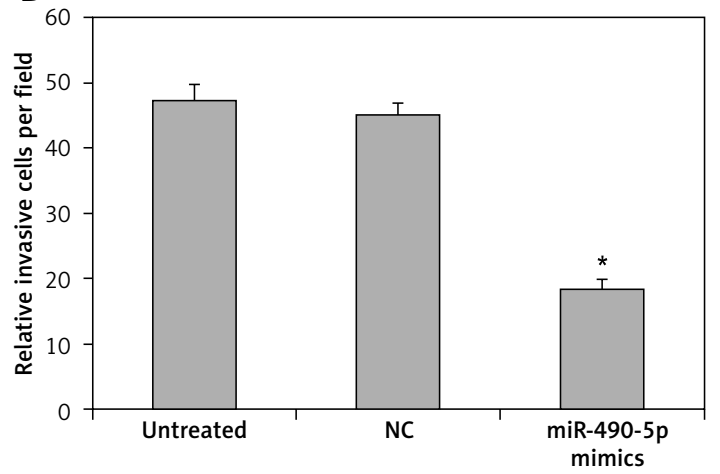

Figure 3. Effects of miR-490-5p overexpression on cell proliferation, apoptosis and invasion activity in T24 cell line in vitro. $\mathbf{A}$ - Expression level of miR-490-5p in the untreated group, the negative control (miRNA) group and the miR-490-5p mimics group ( $\left.{ }^{*} p<0.01\right)$. B - Cell viability assayed by MTT assay at $0 \mathrm{~h}, 24 \mathrm{~h}, 48 \mathrm{~h}$ and $72 \mathrm{~h}$ after transfection ${ }^{*} p<0.05$ at $48 \mathrm{~h}$, and ${ }^{* *} p<0.01$ at $72 \mathrm{~h}$ ). C - Caspase- 3 level in T24 cells after miR-490-5p overexpression $\left({ }^{*} p<0.01\right)$. D - Cell invasion ability after miR-490-5p overexpression in T24 cells $\left({ }^{*} p<0.01\right)$ 
A

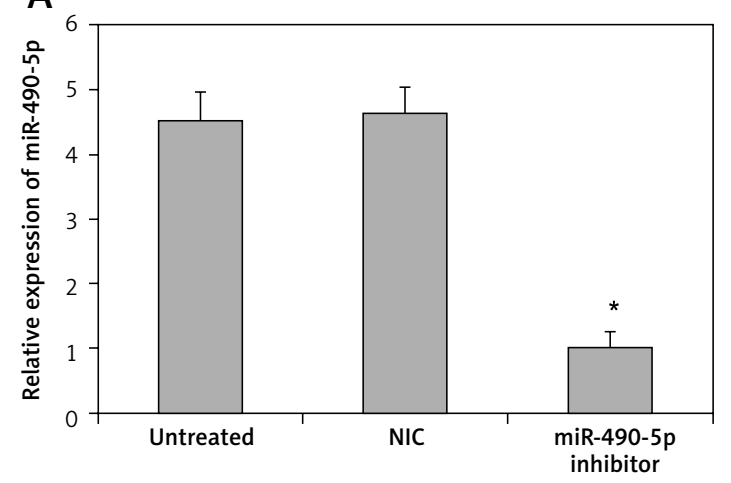

C

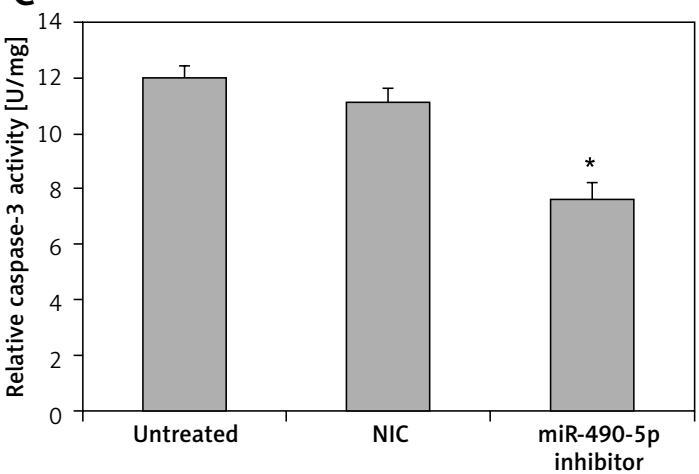

B

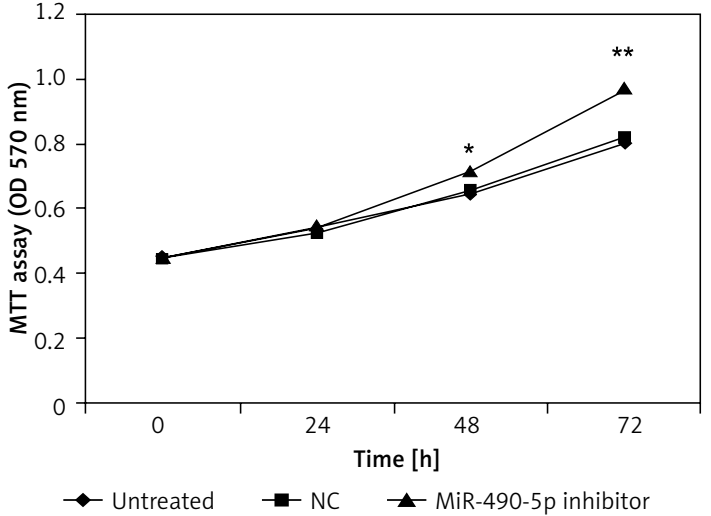

D

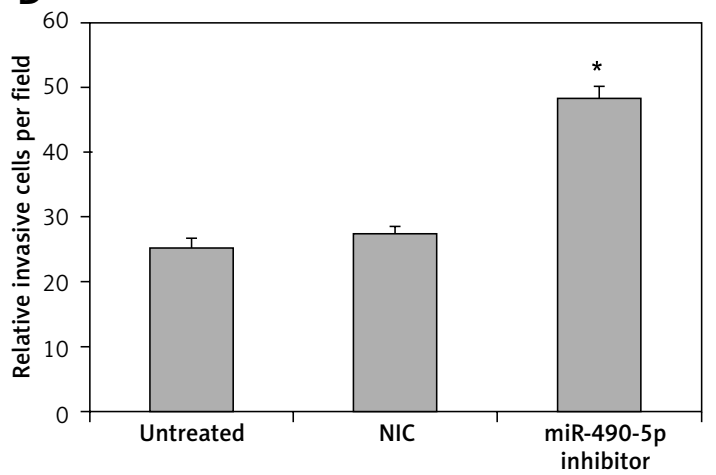

Figure 4. Effects of miR-490-5p suppression on cell proliferation, apoptosis and invasion activity in T24 cell line in vitro. A - Expression level of miR-490-5p in the untreated group, the negative control miRNA group and the miR-490-5p inhibitor group ( $\left.{ }^{*} p<0.01\right)$. B - Cell viability assayed by MTT assay at $0 \mathrm{~h}, 24 \mathrm{~h}, 48 \mathrm{~h}$ and $72 \mathrm{~h}$ after transfection ( ${ }^{*} p<0.05$ at $48 \mathrm{~h} ;{ }^{* *} p<0.01$ at $72 \mathrm{~h}$ ). C - Caspase-3 level in T24 cells after miR-490-5p suppression $\left({ }^{*} p<0.01\right)$. D - Cell invasion ability after miR-490-5p suppression in T24 cells $\left({ }^{*} p<0.01\right)$

The apoptosis activity was evaluated by detecting the activity of caspase-3. As shown in Figure 3 C, the activity of caspase- 3 in the miR-490-5p mimics group was higher than that in the other two groups. This result suggests that miR-490-5p induced cell apoptotic cell death in bladder tumor cells.

Cell invasion was assessed by transwell assay with Matrigel. As shown in Figure $3 \mathrm{D}$, the number of invading cells was significantly decreased in the miR-490-5p transfection group compared with the controls.

Collectively these data demonstrate that overexpression of miR-490-5p inhibited cell proliferation and cell invasion of bladder cancer cells, while it induced cell apoptosis of bladder cancer cells.

\section{Effects of miR-490-5p down-regulation}

on cell proliferation, apoptosis and invasion activity of T24 cells

We transfected T24 cells with miR-490-5p inhibitor and evaluated the effects of miR-490-5p suppression. As shown in Figure $4 \mathrm{~A}$, the expression level of miR-490-5p in the miR-490-5p inhibitor group was significantly lower than in the negative control miRNA group and the untreated group, indicating that the miR-490-5p inhibitor was successfully transfected into the T24 cells.

As shown in Figure $4 \mathrm{~B}$, there were no significant differences among the three groups at $0 \mathrm{~h}$ and $24 \mathrm{~h}$ after transfection. However, at $48 \mathrm{~h}$ and $72 \mathrm{~h}$ after transfection, the miR-490-5p transfected cells exhibited significantly higher growth compared with the negative control cells or the untreated T24 cells.

As shown in Figure 4 C, the activity of caspase-3 in the miR-490-5p inhibitor group was lower than that in the other two groups. Also the number of invading cells was significantly increased in the miR-490-5p transfection group compared with the controls shown in Figure $4 \mathrm{D}$.

The results demonstrate that suppression of miR-490-5p expression promotes cell proliferation and cell invasion of bladder cancer cells, while it inhibits cell apoptosis of bladder cancer cells.

\section{C-FOS is a novel target of miR-490-5p}

We further investigated the effect of miR-490-5p overexpression on the level of c-FOS. Quantitative results of western blotting are shown in Figure $5 \mathrm{~A}$, and representative western blotting results are 
A

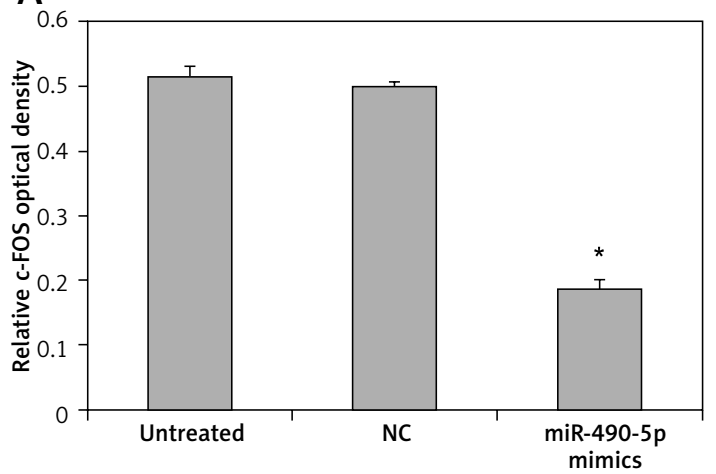

B

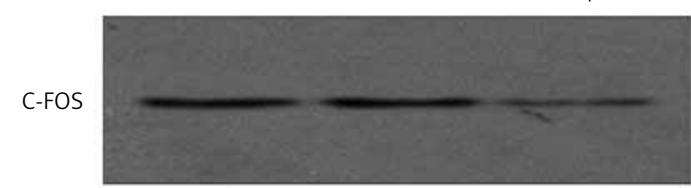

UVRAG

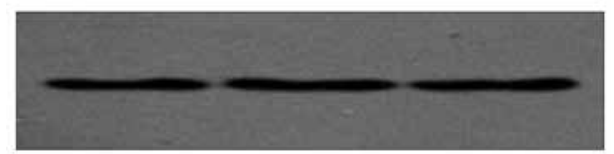

Figure 5. Effects of miR-490-5p overexpression on c-FOS expression in T24 cells. Quantitative results of western blotting are shown in (A) and representative western blotting results are shown in (B). UVRAG was also blotted and served as an internal control

shown in Figure 5 B. The c-FOS protein level in the miR-490-5p mimics group was significantly lower than that in the other two groups. The effect of miR-490-5p suppression on the level of c-FOS was also observed. The c-FOS protein level in the miR-490-5p inhibitor group was significantly higher than those in the other two groups, as shown in Figures $6 \mathrm{~A}, \mathrm{~B}$. These results indicated that miR-490-5p targeted c-FOS and negatively regulated its expression in bladder cancer.

\section{Discussion}

Recently in cancer research, a large amount of evidence have demonstrated that miRNAs are aberrantly expressed in various types of human cancers. Therefore, many researchers have focused on the cancer-associated miRNAs and their specific target genes to uncover their underlying mechanisms [26]. The first findings about altered miRNA expression in bladder cancer were reported in 2007. In the report, they found that 10 miRNAs were up-regulated in bladder cancer [27].

In the present study, we detected the expression of miR-490-5p in 20 pairs of human bladder cancer tissues and adjacent non-tumor tissues,

A

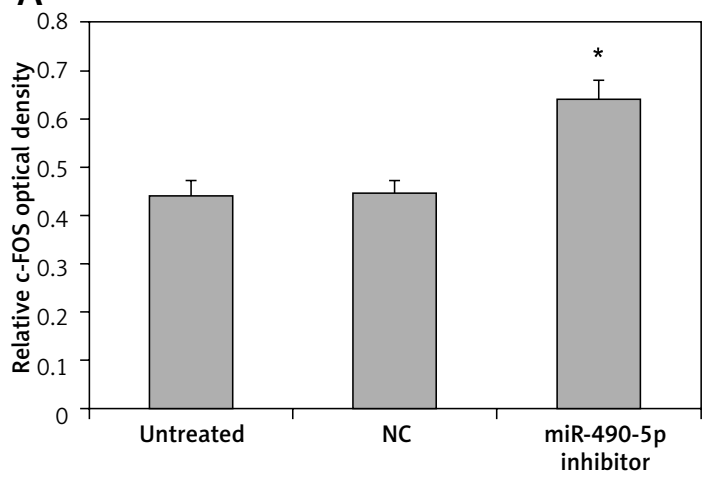

3 bladder cancer cell lines and 1 normal bladder cancer cell line by real-time PCR. The expression of miR-490-5p was found to be significantly decreased in bladder cancer tissues compared to non-tumor tissues, which was in accordance with previous reports $[23,28]$. Moreover, we found for the first time that the expression of miR-490-5p was much lower in bladder cancer cell lines than that in a normal bladder cell line. The down-regulation of miR-490-5p in bladder cancer may contribute to cancer progression and miR-490-5p may act as a tumor suppressor. However, the role of miR-490-5p and its target genes has not been studied in bladder cancer previously. Therefore, in this study, we systematically studied the function and molecular mechanism of miR-490-5p in bladder cancer.

Veerla et al. [29] analyzed 34 bladder cancer cases for miRNA and mRNA expression as well as genomic structure, and found that pathological subtypes of urothelial carcinoma showed distinct miRNA gene expression. Similarly, our analyses showed that there was an inverse relationship between the expression of miR-490-5p and pathological classifications of bladder cancer (Figure $1 \mathrm{C}$ ).

\section{B}

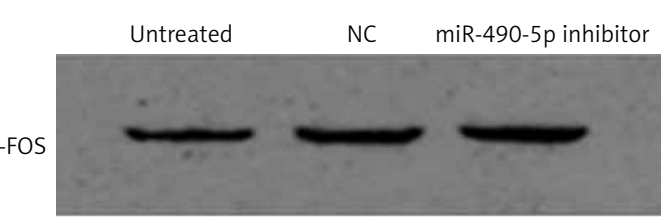

UVRAG

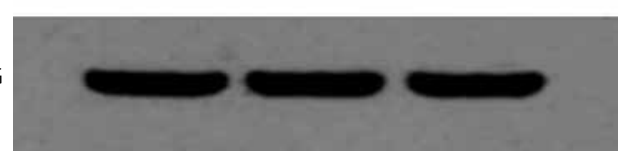

Figure 6. Effects of miR-490-5p suppression on c-FOS expression in T24 cells. Quantitative results of western blotting are shown in (A) and representative western blotting results are shown in (B). UVRAG was also blotted and served as an internal control 
To study the role of miR-490-5p in bladder cancer, we overexpressed and suppressed miR490-5p, and detected the changes in cellular activities in bladder cancer cell line T24. MTT assay showed that the up-regulation of miR-490-5p inhibited cell growth. Transwell assay with Matrigel showed that overexpression of miR-490-5p significantly reduced the invasion of cells. Additionally, caspase-3 was used as a maker for detecting programmed cell death in this study. Caspase-3, which plays a central role in the execution phase of cell apoptosis, is activated in the apoptotic cells by both extrinsic and intrinsic pathways [30]. The results showed that the level of caspase- 3 increased significantly after miR-490-5p over-expression compared to the controls, suggesting that miR-490-5p could promote T24 cell apoptosis. Accordingly, down-regulation of miR-490-5p significantly promoted cell growth and invasion while inhibiting cell apoptosis. All these results indicated the potential tumor suppressive role of miR-490-5p in bladder cancer.

C-FOS is a cellular proto-oncogene belonging to the gene family of transcription factors, which often combines with c-JUN to form the AP-1 transcription factor. C-FOS has been shown to participate in the regulation of cell proliferation, differentiation, apoptosis, and transformation [31]. As previously reported, the level of c-FOS was significantly higher in bladder cancer tissues than those in normal and adjacent non-cancer tissues, suggesting that c-FOS might be involved in the development of bladder cancer [32]. The results of our study were consistent with this report.

According to bioinformatics analysis, c-FOS was supposed to be the target gene of miR-490-5p in the present study. To further validate that, western blotting was conducted. Compared to the other two groups, the c-FOS expression was lower in the group transfected with miR-490-5p mimics and higher in the group transfected with a miR-490-5p inhibitor, indicating that c-FOS was targeted by miR-490-5p.

In conclusion, our present study identified miR490-5p as a tumor suppressor that functioned through targeting c-FOS in bladder cancer. Meanwhile, this study suggests that miR-490-5p could be considered as a potential target for the treatment and management of bladder cancer in the future. A limitation of the study was the relatively small sample size. Thus, further investigation with a larger sample size will be done in the future.

\section{Acknowledgments}

This study was supported by Hunan province science supporting grant no. $20010 N F 3716$.

\section{Conflict of interest}

The authors declare no conflict of interest.

\section{References}

1. Jemal A, Bray F, Center MM, Ferlay J, Ward E, Forman D. Global cancer statistics. CA Cancer J Clin 2011; 61: 69-90.

2. Kakizoe T, Mucci LA, Albertsen PC, Droller MJ. Screening for bladder cancer: theoretical and practical issues in considering the treated and untreated natural history of the various forms of the disease. Scand J Urol Nephrol Suppl 2008; 218: 191-212.

3. Donat SM. Integrating perioperative chemotherapy into the treatment of muscle-invasive bladder cancer: strategy versus reality. J Natl Compr Canc Netw 2009; 7: 40-7.

4. Nese N, Gupta R, Bui MH, Amin MB. Carcinoma in situ of the urinary bladder: review of clinicopathologic characteristics with an emphasis on aspects related to molecular diagnostic techniques and prognosis. J Natl Compr Canc Netw 2009; 7: 48-57.

5. Rochester MA, Patel N, Turney BW, et al. The type 1 insulin-like growth factor receptor is over-expressed in bladder cancer. BJU Int 2007; 100: 1396-401.

6. Wang CC, Chiang YM, Kuo PL, Chang JK, Hsu YL. Norsolorinic acid inhibits proliferation of T24 human bladder cancer cells byarresting the cell cycle at the $\mathrm{GO} /$ G1 phase and inducing a Fas/membrane-bound Fasligand-mediated apoptotic pathway. Clin Exp Pharmacol Physiol 2008; 35: 1301-8.

7. May M, Helke C, Nitzke T, Vogler H, Hoschke B. Survival rates after radical cystectomy according to tumor stage of bladder carcinoma at first presentation. Urol Int 2004; 72: 103-11.

8. Aglamis E, Toktas G, Unluer E, et al. Prognostic factors in radical cystectomy affecting survival. Arch Med Sci 2012; 8: 650-4.

9. Kim WJ, Bae SC. Molecular biomarkers in urothelial bladder cancer. Cancer Sci 2008; 99: 646-52.

10. Jabłonowski Z, Reszka E, Gromadzińska J, et al. Hypermethylation of $\mathrm{p} 16$ and DAPK promoter gene regions in patients with non-invasive urinary bladder cancer. Arch Med Sci 2011; 7: 512-6.

11. Chow TF, Mankaruos M, Scorilas A, et al. The miR-17-92 cluster is over expressed in and has an oncogenic effect on renal cell carcinoma. J Urol 2010; 183: 743-51.

12. Visone R, Veronese A, Rassenti LZ, et al. miR-181b is a biomarker of disease progression in chronic lymphocytic leukemia. Blood 2011; 118: 3072-9.

13. Takeshita F, Patrawala L, Osaki M, et al. Systemic delivery of synthetic microRNA-16 inhibits the growth of metastatic prostate tumors via downregulation of multiple cell-cycle genes. Mol Ther 2010; 18: 181-7.

14. Croce CM, Calin GA. miRNAs, cancer, and stem cell division. Cell 2005; 122: 6-7.

15. Bartel DP. MicroRNAs: genomics, biogenesis, mechanism, and function. Cell 2004; 116: 281-97.

16. Croce CM. Causes and consequences of microRNA dysregulation in cancer. Nat Rev Genet 2009; 10: 704-14.

17. Lim LP, Lau NC, Garrett-Engele P, et al. Microarray analysis shows that some microRNAs downregulate large numbers of target mRNAs. Nature 2005; 433: 769-73.

18. Chen CZ. MicroRNAs as oncogenes and tumor suppressors. N Engl J Med 2005; 353: 1768-71.

19. Dyrskjot L, Ostenfeld MS, Bramsen JB, et al. Genomic profiling of microRNAs in bladder cancer: miR-129 is associated with poor outcome and promotes cell death in vitro. Cancer Res 2009; 69: 4851-60.

20. Noguchi S, Mori T, Hoshino Y, et al. MicroRNA-143 functions as a tumor suppressor in human bladder cancer T24 cells. Cancer Lett 2011; 307: 211-20. 
21. Hirata H, Hinoda Y, Ueno K, Shahryari V, Tabatabai ZL, Dahiya R. MicroRNA-1826 targets VEGFC, beta-catenin (CTNNB1) and MEK1 (MAP2K1) in human bladder cancer. Carcinogenesis 2012; 33: 41-8.

22. Yamada Y, Enokida H, Kojima S, et al. MiR-96 and miR183 detection in urine serve as potential tumor markers of urothelial carcinoma: correlation with stage and grade, and comparison with urinary cytology. Cancer Sci 2011; 102: 522-9.

23. Han Y, Chen J, Zhao X, et al. MicroRNA expression signatures of bladder cancer revealed by deep sequencing. PLoS One 2011; 6: e18286.

24. Schmittgen TD, Lee EJ, Jiang J, et al. Real-time PCR quantification of precursor and mature microRNA. Methods 2008; 44: 31-8.

25. Griffiths-Jones S, Grocock RJ, van DS, Bateman A, Enright AJ. miRBase: microRNA sequences, targets and gene nomenclature. Nucleic Acids Res 2006; 34 (Database issue): D140-4.

26. Wilmott JS, Zhang XD, Hersey P, Scolyer RA. The emerging important role of microRNAs in the pathogenesis, diagnosis and treatment of human cancers. Pathology 2011; 43: 657-71.

27. Gottardo F, Liu CG, Ferracin M, et al. Micro-RNA profiling in kidney and bladder cancers. Urol Oncol 2007; 25 387-92.

28. Meiri E, Levy A, Benjamin H, et al. Discovery of microRNAs and other small RNAs in solid tumors. Nucleic Acids Res 2010; 38: 6234-46.

29. Veerla S, Lindgren D, Kvist A, et al. MiRNA expression in urothelial carcinomas: important roles of miR-10a, miR222, miR-125b, miR-7 and miR-452 for tumor stage and metastasis, and frequent homozygous losses of miR-31. Int J Cancer 2009; 124: 2236-42.

30. Ghavami S, Hashemi M, Ande SR, et al. Apoptosis and cancer: mutations within caspase genes. J Med Genet 2009; 46: 497-510.

31. Durchdewald M, Angel P, Hess J. The transcription factor Fos: a Janus-type regulator in health and disease. Histol Histopathol 2009; 24: 1451-61.

32. Yao HQ, Peng Y, Zhong ZZ, He HX, Li ZH. Association of the expressions of platelet-derived growth factor receptor and c-Fos with the biological characteristics of bladder cancer. Di Yi Jun Yi Da Xue Xue Bao 2004; 24: 177-9. 